\section{0-316 TRANSFORMATIONAL LEADERSHIP AND FIREFIGHTERS' WELL-BEING: A STUDY WITH EMERGENCY TEAM PROFESSIONALS}

'Mariana Neto, Maria José Chambel, Luis Alberto Curral, Daniel Dominguez, Sónia Namorado, Raquel Pinheiro, Vitor Reis, Joana Faria-Anjos, Rui Ângelo. 'National Institute of Health Dr. Ricardo Jorge, Portugal

\subsection{6/OEM-2021-EPI.159}

Introduction Although research on leadership has privileged its effect on performance, there is also some empirical evidence that leadership can have a beneficial effect on the well-being of subordinates. The analysis of this effect in professions with high demands that can affect stress and well-being, as is the case with firefighters, is especially relevant.

Objectives In this study based on the theory of transformational leadership, we considered that with this leadership style, subordinates had more resources to face the situations of high demands that characterize their profession. In this way, we analyzed the effect of leadership on well-being, namely exhaustion and flourishing.

Method With a sample of 90 firefighters from emergency intervention teams, in T1 we assessed transformational leadership and in T2, about 3-4 weeks later we assessed well-being. In this period, we recorded the daily number of critical incidents in which each firefighter intervened.

Results The results obtained allowed us to observe that the different dimensions of transformational leadership were significant predictors of both exhaustion and flourishing and this effect occurred after controlling for the number of critical incidents.

Conclusion There must be tools that invest in the development of transformational leadership skills of the heads of firefighters, in order to promote a healthy work context for these professionals.

\section{0-488 ASSESSMENT OF MULTIDISCIPLINARY INTERVENTION OF OCCUPATIONAL MEDICINE AND PHYSIOTHERAPY ON RETURN TO WORK OF PATIENTS WITH MUSCULOSKELETAL DISORDERS}

${ }^{1}$ Talal Ibrahim, Ronit Calderon-Margalit, Lilah Rinsky Halivni. 'Clalit hmo, Israel

\subsection{6/OEM-2021-EPI. 160}

Introduction Musculoskeletal disorders (MSD), are a major public health concern worldwide, leading to substantial individual and societal burdens, incurring disability, work incapacitation, and absenteeism. Multidisciplinary interventions focusing on rehabilitating MSD are generally effective in facilitating integration and return-to-work (RTW). Israel serves as an ideal milieu for conducting multidisciplinary interventions, because OM service is free, egalitarian, and nationally provided to every worker by four Health Maintenance Organizations (HMOs), comparable to the physiotherapy (PT) service. Objectives Evaluating the effectiveness of a joint OM and PT intervention program in terms of RTW rates and time to RTW.

Methods A quasi-experiment was conducted among MSD patients referred to the OM clinic of Jerusalem District's Clalit HMO between 1/2016 and 6/2018. Patients allocated to OM-OT multidisciplinary intervention were matched to usual- care OM patients, receiving uncoordinated OT. Work status was followed-up using electronic medical records, employers' communication, and phone interviews in 1/2019 assessing RTW. Multivariate adjusted regression and survival analyses were performed to evaluate associations between the intervention and RTW rates or time to RTW, including stratified analyses for symptom anatomy.

Results Of 249 patients included in the study, 94 (38\%) were treated by the multidisciplinary OM-OT intervention which demonstrated higher proportions of full-RTW (71.2\%) compared to the usual care $(51.6 \%)$. The intervention proved effective in RTW $(\mathrm{OR}=2.6, \mathrm{CI}=1.4-4.8)$, which was earlier $(\mathrm{HR}=1.7, \mathrm{CI}=1.2-2.4)$, with a median time to full-RTW of three months $(\mathrm{SE}=0.44)$ in the intervention group versus nine $(S E=6.6)$ in the usual-care group. Higher odds for RTW were demonstrated among intervention patients with back/neck symptoms (3.3) compared to limbs (1.9).

Conclusions Our field study proves the effectiveness of OMOT collaboration in RTW, coordinating existing public medical systems that provide holistic treatment approaches. OM physicians' involvement assists in tailoring rehabilitation OT treatment towards occupational-oriented goals. Additional multidisciplinary collaborations which include psychotherapists, occupational therapists, etc. should be further studied.

\section{Poster Presentations}

\section{Poster}

\section{\begin{tabular}{l|l} 
P-6 & DIFFERENCES IN WORK ENVIRONMENTS DURING
\end{tabular} PREGNANCY WERE RELATED TO POSTPARTUM RETURN TO WORK: A POPULATION-BASED COHORT STUDY}

${ }^{1}$ Ping Shih, Tung-liang Chiang, Yue Leon Guo. 'National Taiwan University Hospital, Taiwan

\subsection{6/OEM-2021-EPI. 161}

Introduction The female labor force participation rate is increasing or has remained in high proportion in many countries, and there has been a marked rise in the participation of women with infants in the labor market. In Taiwan, an effort was made to make the workplace friendly for females, especial for mothers-to-be and breastfeeding mothers, under the legal protection. However, some women could not successfully return to work (RTW) after giving birth right after maternity leave.

Objective This study aimed to explore the possible risk factors in the work environment associated with maternal postpartum RTW.

Methods We used the Taiwan Birth Cohort Study, recruiting representative mother-infant pairs using multistage stratified sampling. Participating mothers underwent two home interviews at 6 and 18 months after delivery using structured questionnaires. Mothers actively employed at the beginning of their pregnancy were included. Self-reported 5-point severity gradation of job stress pre- and during pregnancy and time of RTW after childbirth were recorded. Multiple logistic regression analysis was performed to calculate adjusted odds ratios $(\mathrm{aOR})$ and 95\% confidence intervals (CI). 
Results 9,369 women returning to the same job within 1 year after delivery were included. Among them, 8,478 (90.5\%) mothers returned to work after no more than 2 months, when they would be assumed to have a full-paid maternity leave. Compared with those having stationary workload, those suffering from increased job stress during pregnancy had a 1.72 times greater risk of later RTW - between 6 and 12 months, after adjusting for potential confounders. Moreover, among those not returning to work more than 2 months after delivery, the aOR of later RTW was 2.0 (95\% CI: 1.25, 3.19) among those with increased job stress.

Conclusions In this prospective birth cohort study, mothers' prenatal work conditions were significantly related to later RTW for those returning to work within 1 year after childbirth.

\section{P-7 WHICH ARE THE DETERMINANTS INFLUENCING THE INTENSION OF NURSES TO STAY AT THEIR INSTITUTION}

${ }^{1}$ Miho Satoh. 'Yokohama City University, Japan

\subsection{6/OEM-2021-EPI.162}

Objective This study aimed to investigate the main determinants related to their intension to stay at an institution, by years of clinical experience, among nurses in Japan.

Method A cross-sectional survey was conducted at 12 hospitals in the Tohoku and Kanto regions of Japan. Of the 1,034 nurses working in those hospitals, 481 nurses (response rate: $46.5 \%$ ) completed a questionnaire and contributed to the analyses. The participants identified the determinants strengthening their intention to stay at the current hospital ('comfortable workplace environment,' 'passive motivational factors,' 'convenience of hospital location,' 'favorable worklife balance,' and 'fulfilment in nursing'), and individual attribution. The radar charts were shown to assess the determinants strengthening the intention to stay based on the standardized scores of determinants strengthening the intention to stay.

Results Nurses having less than 9 years of clinical experience and having 10-19 years of clinical experience showed higher scores of 'passive motivational factors' than the scores for the other determinants. Nurses having less than 9 years of clinical experience rated the lowest scores for 'favorable work-life balance' among all the determinants. On the other hand, nurses having more than 20 years of clinical experience gave the highest scores to 'favorable work-life balance', and the lowest scores to 'passive motivational factors.' Nurses having less than 9 years of clinical experience gave lower scores to 'comfortable workplace environment,' 'convenience of hospital location,' 'favorable work-life balance,' and 'fulfilment in nursing' than the other nurses. Nurses having more than 20 years of clinical experience showed opposite response trends compared to nurses having less than 9 years of clinical experience.

Conclusion Which determinants nurses emphasized in relation with their intention to stay would depend on the duration of clinical experience. Nurses having less than 9 years of clinical experience would be likely to stay at their current institution with passive motivation.

\section{P-9 THE COMBINED EFFECTS OF A HIGH PHYSICAL WORKLOAD AND EITHER OVERWEIGHT/OBESITY OR INSUFFICIENT VIGOROUS PHYSICAL ACTIVITY ON SELF- RATED HEALTH}

${ }^{1}$ Karin Proper, Mandy van den Berge, Sandra van Oostrom, Henk Van der Molen, Suzan Robroek, Carel Hulshof, Allard van der Beek. 'National Institute for Public Health and the Environment, Netherlands

\subsection{6/OEM-2021-EPI.163}

Introduction High physical workload and unhealthy lifestyle behaviors are common among blue-collar workers, both being separate risk factors for self-rated health. It may however be that the combination of high physical workload and an unhealthy lifestyle have a stronger effect than the sum of these factors, but studies to the combined effects are scarce. Objective To investigate the combined effects of a high physical workload and either overweight/obesity or insufficient vigorous physical activity on self-rated health.

Methods A longitudinal study was performed among 29,987 construction workers using data of two Workers\&rsquo; Health Surveillance Programs. Self-reported physical workload involved strenuous work postures and manual material handling. Insufficient vigorous physical activity was defined as self-reported vigorous activity for less than 3 times per week. Overweight/obesity was measured by physical examination. Self-rated health was measured using a single item question. Logistic regression analysis was used to investigate the associations between the separate risk factors at baseline and selfrated health at follow-up. The combined effects of physical workload and overweight/obesity or insufficient vigorous activity on self-rated health were analyzed using the relative excess risk due to interaction (RERI).

Results Construction workers with strenuous work postures (OR 1.35 95\%CI 1.25-1.46) or manual material handling (OR 1.29 95\%CI 1.19-1.40) were more likely to report poor self-rated health at follow-up. Overweight was not associated with poor self-rated health at follow-up, but obesity (OR 1.31 95\% CI 1.17-1.47) and insufficient vigorous activity (OR 1.13 95\%CI 1.01-1.25) were. However, no statistically significant interaction effects were found for physical workload and obesity or insufficient vigorous activity.

Conclusions Physical workload, obesity and insufficient vigorous activity were separate risk factors for poor self-rated health, but did not appear to have a synergistic effect. Worksite health promotion interventions focusing on improvement in physical workload and lifestyle both can have beneficial health effects.

\section{P-13 THE UTILITY OF OCCUPATIONAL HEALTH DATA IN THE CANADIAN PARTNERSHIP FOR TOMORROW'S HEALTH (CANPATH)}

${ }^{1}$ Ellen Sweeney, Philip Awadalla, Parveen Bhatti, Philippe Broet, Trevor Dummer, John McLaughlin, Donna Turner, Jennifer Vena. 'Dalhousie University, Canada

\subsection{6/OEM-2021-EPI.164}

Introduction The Canadian Partnership for Tomorrow's Health (CanPath) is a multi-centered prospective cohort study, and represents Canada's largest population health research 